\title{
Cure temperature influences composite electrical properties by carbon nanotube-rich
} domain formation ${ }^{\dagger}$

\author{
Chelsea S. Davis ${ }^{1}$, Nathan D. Orloff ${ }^{1}$, Jeremiah W. Woodcock ${ }^{1}$, Christian J. Long ${ }^{2,3}$,
}

Kevin A. Twedt ${ }^{2,3}$, Bharath Natarajan ${ }^{2,3}$, Jonathan E. Seppala ${ }^{1}$, Jabez J. McClelland ${ }^{2}$, Jan Obrzut $^{1}$, J. Alexander Liddle ${ }^{2}$, Jeffrey W. Gilman ${ }^{1 *}$

${ }^{1}$ Material Measurement Laboratory, National Institute of Standards and Technology, Gaithersburg, Maryland, USA

${ }^{2}$ Center for Nanoscale Science and Technology, National Institute of Standards and Technology, Gaithersburg, Maryland, USA

${ }^{3}$ Maryland Nanocenter, University of Maryland, College Park, Maryland, USA

${ }^{*}$ Corresponding author: Jeffrey W. Gilman E-mail: jeffrey.gilman@nist.gov, tel:

301.975.6573.

'Official contribution of the National Institute of Standards and Technology (NIST); not subject to copyright in the United States. The full description of the procedures used in this article requires the identification of certain commercial products and their suppliers. The inclusion of such information should in no way be construed as indicating that such products or suppliers are endorsed by NIST or are recommended by NIST or that they are necessarily the best materials, instruments, software or suppliers for the purposes described. 


\begin{abstract}
Carbon nanotube (CNT) nanocomposites are enticing materials that enable engineers to tailor structural and electrical properties for applications in the automotive and aerospace industries. CNT mass fraction and the matrix cure temperature are two ways to tune the direct and alternating current electrical properties of these nanocomposites; yet, how mass fraction and cure temperature affect electrical properties remains unclear. In many cases, nanofillers such as carbon nanotubes appear in concentrated domains within the nanocomposite. Recent insights into nanoparticle-rich domain formation and its influence on electrical properties raise questions about which processing variables might optimally tune a material's functionality. Utilizing recently developed, nondestructive measurement techniques such as scanning lithium ion microscopy and microwave cavity perturbation, new insights are presented into the role of mass fraction and cure temperature in multiwall carbon nanotube - bisphenol A diglycidyl ether epoxy composites. Here, it is found that both mass fraction and cure temperature affect the electrical properties. Specifically, beyond the electrical percolation threshold, the DC conductivity is an order of magnitude higher for composites prepared at elevated cure temperatures for a given CNT mass fraction. Direct observations of the microdomain morphology do not show substantial differences due to cure temperature. These findings suggest pathways to generate designer nanocomposites for advanced electrically-active applications.
\end{abstract}

\title{
Keywords
}

Carbon nanotubes, nanocomposites, electrical properties, non-destructive testing, scanning ion microscopy

\section{Introduction}

Multi-walled carbon nanotube (MWCNT) reinforced polymer nanocomposites are both scientifically and industrially relevant due to the unique combination of electrical and structural properties observed at low filling fractions.[1] The formation of carbon nanotube (CNT)-rich domains (simply "domains" throughout this text) within these nanocomposite materials has routinely been observed in manufactured nanocomposites.[2] The role that such domains play in the overall electrical performance relative to a perfectly dispersed mixture of MWCNT is particularly interesting.[3-5] Hence, understanding the influence of domain morphology on MWCNT nanocomposite electrical properties will enable materials 
engineers to minimize MWCNT mass fraction through control of manufacturing processes while tuning overall electrical performance. This control will facilitate the development of new materials for electromagnetic shielding,[6,7] electrostatic discharge protection,[8] and electrically active structural applications.[9]

Numerous reports have shown that tuning the amount of nanotubes within the polymer matrix affects electrical properties of MWCNT nanocomposites.[10-18] Many research efforts have focused on achieving individually dispersed nanotube morphologies to form three dimensional percolating networks to enhance electrical conductivity.[19,20] Further, it has recently been suggested that perhaps the most well dispersed system is not necessarily the most electrically useful one.[21-23] It has further been suggested that a certain degree of MWCNT aggregation might be desirable to achieve higher electrical conductivity.[16,21,24,25] To this end, experimental techniques have sought to control the degree of aggregation in MWCNT nanocomposites.[26] These dispersion techniques range from mechanical processing methods (e.g., high shear mixing,[27] calendering,[28] sonication[29,30]) to surface functionalization[10,31-33] of the carbon nanotubes themselves. The ability to quantify the electrical properties of electrically conducting nanocomposites is critical. In most cases, the conductivity and the dielectric constant quantify the electrical performance and the impact of processing variables including MWCNT concentration. Typically, two-point or four-point[34] probe measurements are utilized to measure direct current (DC) behavior. $[35,36]$ Recent advances in nondestructive, noncontact alternating current (AC) microwave perturbation methods have also been implemented to characterize AC behavior.[37] Here, MWCNT concentration and matrix cure temperature were used to tailor the microstructure and thereby tune the electrodynamics of the bulk nanocomposite. The corresponding microstructure, specifically the domain size and dispersion quality, were measured and correlated to changes in the electrical properties.

In the following investigation, samples were comprised of various concentrations of thoroughly sonicated and shear mixed MWCNT in an epoxy network, cured at either $\mathrm{T}_{\mathrm{c}}=25^{\circ} \mathrm{C}$ or $\mathrm{T}_{\mathrm{c}}=80^{\circ} \mathrm{C}$. The DC and AC conductivity of the nanocomposites were measured to determine correlations between the MWCNT mass fraction, cure temperature and electrical properties. Two-point and four-point probe techniques were used to measure the DC conductivity.[34,38] The AC electrical conductivity was characterized by a novel, nondestructive microwave cavity perturbation measurement, following the partial filling technique for high conductivity and high dielectric constant materials.[37] Next, a detailed 
nanostructure characterization by scanning lithium ion microscopy (SLIM) was performed.[39] Image analysis methods were then applied to quantify the microstructure of the nanocomposite and determine how the dispersion quality changes as a function of mass fraction and cure temperature. By quantifying the dispersion as a function of mass fraction and curing temperature, the role that microstructure (specifically domain size and spacing) plays in controlling electrical properties was explored.

\section{Experimental Methods ${ }^{\dagger}$}

\subsection{Sample Preparation}

MWCNT and polyetherdiamine epoxy nanocomposites were prepared at various filler loadings. The MWCNT (Graphistrength C100 (R\&D), Batch 110314, Lot 005) used here were obtained as a black powder from Arkema (King of Prussia, PA). A transmission electron microscope (TEM) was employed to measure MWCNT geometry (average diameter $=31 \mathrm{~nm} \pm 7 \mathrm{~nm}$, average length $=302 \mathrm{~nm} \pm 164 \mathrm{~nm}$ ) (unless otherwise noted, all uncertainties are one standard deviation taken from a number of measurements). The MWCNT were used as received and had a nominal mass fraction of less than $12 \%$ catalyst (referred to as "mass\%" throughout this work, aluminum oxide $\leq 7$ mass $\%$ and iron oxide $\leq$ 5 mass $\%$ ). The powdered material was stored in an opaque polypropylene container at room temperature. The matrix was a commonly employed epoxy/polyetherdiamine system. An epoxy-functionalized monomer of bisphenol A diglycidyl ether (DGEBA, D.E.R. ${ }^{\text {TM }}$ 332, Sigma Life Science, St. Louis, MO) and a curing agent mixture containing two polyetherdiamines (Huntsman Jeffamines D-230 and D-2000) was used to enhance epoxy toughness.

First, MWCNT were added to the Jeffamine mixture (9:1 D-230:D-2000 by mass) to achieve a 1.00 mass $\%$ MWCNT concentration in the final epoxy formulation. The Jeffamine/MWCNT mixture was placed in a high shear mixer (FlackTek, Inc. SpeedMixer ${ }^{\mathrm{TM}}$ DAC $150 \mathrm{SP}$, Landrum, SC) at $365 \mathrm{rad} / \mathrm{s}$ for $5 \mathrm{~min}$ to achieve an initial coarse dispersion of the MWCNT in the polyetheramine mixture. The dispersion was further mixed with a sonic probe (Ultrasonic Processor GE-50, Cole-Palmer) for six cycles of 5 min sonication followed by $5 \mathrm{~min}$ rest to prevent overheating (30 min total sonication time). The sonicator was fitted with a flat $6.4 \mathrm{~mm}$ diameter probe and had a maximum power output of $750 \mathrm{~W}$ at an acoustic frequency of $20 \mathrm{kHz}$.

Davis et al. 
Next, lower MWCNT concentration Jeffamine mixtures were prepared through serial dilution of the 1.00 mass\% stock dispersion by adding more Jeffamine curing agent (9:1 D230:D-2000 by mass). The DGEBA monomer was added to each dispersion to attain a fully crosslinked epoxy network upon completion of curing. The MWCNT/curing agent/monomer mixture was placed in the high shear mixer at $365 \mathrm{rad} / \mathrm{s}$ for $20 \mathrm{~min}$ and then degassed in a vacuum oven (13 Pa, Fisher Scientific Isotemp Model 281A) at $80{ }^{\circ} \mathrm{C}$ for $10 \mathrm{~min}$.

Finally, the degassed dispersion was slowly poured into a silicone mold on a level surface with care being taken to avoid the introduction of gas bubbles (Figure 1a). The epoxy was cured at either $\mathrm{T}_{\mathrm{c}}=25^{\circ} \mathrm{C}$ or $\mathrm{T}_{\mathrm{c}}=80^{\circ} \mathrm{C}$ for $48 \mathrm{~h}$. The resulting solid nanocomposite coupons were utilized for subsequent electrical conductivity and morphological characterization experiments.

\subsection{Electrical Conductivity Characterization}

The DC and AC electrical conductivities of the MWCNT nanocomposites were measured. DC conductivity was measured with a standard four-point probe station that uses two outer electrodes to apply a current through the material and measures the voltage drop between two inner electrodes (Figure 1d).[34] For mass fractions below 0.30 mass $\%$, the composite resistivity was too high for the four-point probe technique, so a two-point probe test fixture was used in conjunction with an ohm-meter. This measurement method substitution was made because the resistance of the four-point probe voltmeter becomes comparable to the resistance of the nanocomposite sample at low MWCNT concentrations. As such, a two-point technique is more accurate for high dielectric materials. The sheet resistance was then computed and divided by the sample thickness to compute the DC conductivity of the samples as a function of mass fraction.

Since the DC conductivity is dominated by domain-to-domain contacts, [40] the AC conductivity was also measured with a novel microwave cavity perturbation technique developed by Orloff et al.[37] Unlike the DC techniques, this approach is sensitive to the intrinsic properties of the domains themselves (i.e. tube-tube connectivity).[41,42] In this technique, the sample is systematically inserted into a slot in the cavity, thereby shifting the resonance frequency and quality factor of the cavity. By measuring the shifts in the resonance frequency and quality factor as a function of sample insertion, the AC conductivity can be measured with increased accuracy and precision compared to conventional cavity 
perturbation techniques.[37] For further details on the microwave cavity perturbation measurement method, the reader is refered to Orloff et al.[37] A schematic of the microwave cavity testing setup is provided in the Supporting Information.

In these experiments, $\mathrm{AC}$ conductivity can be derived from Ohm's Law and Maxwell's equations as,

$$
\sigma_{A C}=\sigma_{D C}+2 \pi f \epsilon_{i}
$$

where $\sigma_{D C}$ is the DC conductivity, $f$ is the frequency of operation, and $\epsilon_{i}$ is the dielectric loss.[43] At low frequencies, $\sigma_{A C}$ limits to the DC conductivity, but at high frequencies the dielectric loss dominates. If there is dielectric loss in a system, then the AC conductivity will be larger than the DC conductivity. Dielectric loss is also usually an indicator of elelctrical relaxation processes. For a MWCNT epoxy sample consisting of a distribution of domains, dielectric loss is related to several domain properties (concentration, shape, conductivity, intefracial effects, etc.) and the dielectric loss of the host medium.

\subsection{Curing Kinetics of Epoxy}

Parallel plate shear rheology experiments were used to monitor the viscosity of the epoxy during curing at both cure temperatures $\left(\mathrm{T}_{\mathrm{c}}=25^{\circ} \mathrm{C}\right.$ and $\left.\mathrm{T}_{\mathrm{c}}=80{ }^{\circ} \mathrm{C}\right)$. A small amount of freshly mixed and degassed uncured neat epoxy (formulation described in Section 2.1) was deposited between disposable aluminum plates with a diameter of $25 \mathrm{~mm}$ and the top plate was lowered to a nominal gap of $1 \mathrm{~mm}$. The temperature was controlled with a convection oven and monitored by thermocouples over the course of the experiment. Samples were placed under steady shear at $300 \mathrm{~s}^{-1}$. Once the viscosity increased to $1.5 \mathrm{~Pa}-\mathrm{s}$, an oscillatory shear of $\gamma=10 \%$ was applied at $10 \mathrm{rad}^{-1} \mathrm{~s}^{-1}$. The test was stopped once the shear storage modulus, G', exceeded 36,000 Pa.

\subsection{Composite Morphology Imaging}

To characterize the morphology of the MWCNT-rich domains, each sample was sectioned (Figure 2a) and imaged at relatively low magnification. The objective was to observe the microstructure (size and aggregation) of the micrometer-size domains rather than the individual MWCNT. Charging contrast imaging was used in scanning lithium ion microscopy to image the distribution of the MWCNT-rich domains within the epoxy matrix. In this technique, incident ions positively charge the non-conductive epoxy, preventing the re- 
lease of secondary electrons and causing the epoxy regions to appear dark.[39] The nanotube-rich regions appear bright if they have a conductive path to ground, allowing for the neutralization of the deposited ion charge and the release of secondary electrons. Figure 2 shows the SLIM sample preparation process. A microtome was used to slice $100 \mathrm{~nm}$ thick sections, which were then placed on a flat conductive substrate (gold-coated mica). The thin sample ensured that every MWCNT domain (with a diameter of greater than $100 \mathrm{~nm}$ ) had a conductive path to ground and could be readily identified in the image. Ion images were taken using a custom focused lithium ion beam system that uses a new type of high brightness ion source based on photo-ionization of laser-cooled lithium atoms.[39] Similar image contrast can be obtained in low voltage $(<500 \mathrm{eV})$ electron microscopy, but ion microscopy is less affected by defects in the surface topography and produces images that enable facile image analysis.[44] Comparable transmission electron micrographs (TEM) are provided in the Supporting Information (see Figure S 1 and Figure S 2) as well as a more detailed description of the SLIM technique. For additional information on the SLIM imaging technique, the reader is refered to the work of Twedt et al.[39]

\section{Results and Discussion}

\subsection{Electrical Conductivity}

It has been shown in prior studies that the conductivity of a MWCNT nanocomposite depends on the quality and quantity of nanotubes in the material as well as on the electrically active connections between the nanotubes.[14-17] An overview of the electrical properties as a function of mass fraction shows a positive correlation for both $\mathrm{AC}$ and $\mathrm{DC}$ conductivity. As expected, the conductivity was found to increase as the mass fraction of MWCNT increased (Figure 3). More interestingly, it was observed that the DC electrical conductivity after percolation was reached was an order of magnitude greater for nanocomposites cured at $\mathrm{T}_{\mathrm{c}}=80{ }^{\circ} \mathrm{C}$ than for samples prepared at $\mathrm{T}_{\mathrm{c}}=25^{\circ} \mathrm{C}$.

To determine the percolation threshold concentration, $\phi_{v, c}$, the mass fraction $\%$ was first converted to a volume fraction \% through the simple scaling relationship,

$$
\phi_{v}=\frac{\phi_{m}}{\phi_{m}+\left(1-\phi_{m}\right)\left(\frac{\rho_{C N T}}{\rho_{\text {matrix }}}\right)}
$$


where $\phi_{v}$ is the volume fraction $\%, \phi_{m}$ is the mass fraction $\%$, and $\rho_{C N T}$ and $\rho_{\text {matrix }}$ are the densities of the MWCNT and the epoxy matrix, respectively (here, values of $\rho_{C N T}=$ $1.3 \mathrm{~g} \mathrm{~mL}^{-1}$ and $\rho_{\text {matrix }}=1.1 \mathrm{~g} \mathrm{~mL}^{-1}$ were used). The DC conductivity data was then fit with the exponential function,

$$
\sigma_{D C}=\sigma_{c} \cdot\left(\phi_{v}-\phi_{v, c}\right)^{t}
$$

where $\sigma_{\mathrm{c}}$ is the saturated DC conductivity and the critical exponent was $t_{\left(T_{C}=25^{\circ} \mathrm{C}\right)}=$ $(1.195 \pm 0.633)$, and $t_{\left(T_{C}=80^{\circ} C\right)}=(1.642 \pm 0.794) .[45,46]$ The uncertainty values represent a $95 \%$ confidence interval. The fits are represented by dashed lines in Figure 3a and the fitting parameter values are given in Table 1 . For both data sets, $\phi_{v, c}$ was reached at a MWCNT volume fraction below the lowest concentration measured here of 0.084 volume fraction \%. After electrical percolation was achieved, the DC conductivity of nanocomposites cured at different temperatures differed by an order of magnitude.

The AC conductivity data was fit with a logistic growth model,

$$
\sigma_{A C}=\sigma_{C}^{A C} \cdot\left(1+e^{-r\left(\phi_{v}-\phi_{v, c}^{A C}\right)}\right)^{-1}
$$

where $\sigma_{A C}$ is the AC conductivity, $\phi_{v}$ is the volume fraction $\%, \phi_{v, c}^{A C}$ is the AC threshold concentration, $\sigma_{C}^{A C}$ is the saturated AC conductivity, and $r$ is steepness of the saturation. The models are represented by dashed lines in Figure $3 \mathrm{~b}$ and the fitting parameter values are given in Table 1. For these measurements, Eq. (3) did not effectively model the AC conductivity. Instead we used a purely phenomenological logistic growth model. It is reasonable to expect behavior qualitatively similar to logistic growth as one might expect exponential growth with saturation as volume fraction changes from pure epoxy to a percolated network of MWCNTs in an epoxy matrix. Although not physical, this model does allow quantitative comparisons to be made between the two cure temperatures, and allows for facile comparison with external studies. For the present system, it was found that the differences in the threshold concentration and the steepness were small compared to their associated uncertainties. Meanwhile, saturated AC conductivity increased by $17 \%$ when the cure temperature for the nanocomposites was increased from $\mathrm{T}_{\mathrm{c}}=25^{\circ} \mathrm{C}$ to $\mathrm{T}_{\mathrm{c}}=80^{\circ} \mathrm{C}$.

Considering the differences between the two cure temperatures, a substantion increase in the dielectric loss compared to the DC conductivity (Figure 3c) can be observed. This difference increased with increasing volume fraction. Because the 0 mass $\%$ sample shows no difference in the AC conductivity and the MWCNT electrical properties do not change

Davis et al. 
in this temperature range, the difference could likely be ascribed to the dispersion of the MWCNT, which is reflected in the dielectric loss. It is well known that dispersion affects the dielectric properties of mixtures,[47] but broadband frequency dependence would be required to fully describe the distribution of domains.[48,49] From this observation, it can be concluded that the AC conductivity depends on cure temperature for these MWCNT nanocomposites.

\subsection{Domain Microstructure and Morphology}

\subsubsection{Domain Imaging}

Leveraging the contrast available through the SLIM technique, two sets of MWCNT composites were imaged: one cured at $\mathrm{T}_{\mathrm{c}}=25^{\circ} \mathrm{C}$ (Figure 4, blue box) and the other at $\mathrm{T}_{\mathrm{c}}=80^{\circ} \mathrm{C}$ (Figure 4, red box). At least five secondary electron images were collected with a field of view of $122 \mu \mathrm{m}$ at spatially separated locations on each sample, sufficient to obtain significant statistics on the MWCNT-rich domain distributions. Magnified, inverted images of the 0.30 mass fraction \% samples at each temperature are also shown in Figure 4 to allow the detail of the images to be observed.

Images obtained by SLIM were processed to quantify the size distribution of the MWCNT-rich domains as well as the quality of the dispersion for the various cure temperature and MWCNT concentration samples. Each image was binarized by applying Otsu's thresholding method[50] with ImageJ (National Institutes of Health) image processing software. The Otsu method was chosen because it is a clustering-based localized binarization technique, particularly appropriate for images of small, semi-discrete "particles" (e.g., light colored MWCNT-rich domains in dark epoxy-rich regions).

SLIM imaging of MWCNT nanocomposites allowed for a direct quantification of the amount of nanofiller in each sample. The MWCNT-rich domain area fraction was directly measured by image analysis of each SLIM micrograph. Through the application of Delesse's principle,[51] a common stereological convention which states that particle area fraction is equivalent to particle volume fraction, the measured MWCNT-rich area fraction was converted to a domain volume fraction. It is critical to note here that this volume fraction is that of the domains and not the volume fraction of the nanofiller. These domains are comprised mostly of epoxy but have a disproportionately high MWCNT concentration relative to the overall amount of nanofiller contained in a given sample. Thus, the measured domain area fraction and corresponding domain volume fraction is high relative to the overall 
sample MWCNT mass fraction (measured) and volume fraction (calculated by Eqn. 3). A linear relationship between the MWCNT mass fraction and the domain volume fraction (Figure 5) confirmed that the SLIM consistently imaged a proportionate area of MWCNTrich domains relative to the actual MWCNT content for each sample.

\subsubsection{Domain Size}

To quantify the domain geometry, the particle analyzer tool in ImageJ was applied to the binarized images to identify individual domains, determine the coordinates of their centroids, and calculate their geometric characteristics (e.g., area, length, width). Thus, the aspect ratio of each domain was measured. The average aspect ratio was close to unity (ARmode $=1.55 \pm 0.29)$ so the particles were approximated as circles (spheres in 3D) to calculate the domain radii from the measured areas. For all of the images, only domains with a radius larger than $250 \mathrm{~nm}$ were included in the analysis. This lower domain size cutoff was chosen in part due to the pixelation of the SLIM images. Images were recorded with a field of view of approximately $122 \mu \mathrm{m}$, which corresponds to an individual pixel width of approximately $120 \mathrm{~nm}$. The domain size cutoff was set to twice the pixel width to avoid complications associated with counting single pixels. Imaging at higher resolution would have required significantly more imaging time to acquire the same number of statistics on the domain distributions. Importantly, the domain size cutoff is larger than the ion-beam spot size, which is approximately Gaussian in distribution with a radius of approximately $50 \mathrm{~nm}$. To ensure that this $250 \mathrm{~nm}$ cutoff did not lead to the elimination of a significant number of individual nanotubes, TEM images were obtrained for several of the samples. TEM images are included in the Supporting Information along with a further explanation of the domain size cutoff (Figure S 3).

The Freedman-Diaconis rule[52] was used to obtain histograms of the domain radii (Figure 6) where the highest and lowest bin values were computed from the interquartile range. Then the distributions were fit with log-normal probability density functions. From each fit, the mode and standard deviation of the domain radius were determined (Figure 7a) as a function of mass fraction. Interestingly, while the number of domains increased substantially with increasing MWCNT loading, the mode domain radius was independent of mass fraction and cure temperature over the range of processing variables tested.

Davis et al. 


\subsubsection{Domain Dispersion}

To characterize the dispersion of the domains with respect to one another, the free space length (FSL, Figure 7b) dispersion quantification algorithm was employed, which is a measure of domain spacing.[53] In brief, the method consists of placing squares of a given dimension randomly over the binarized image. For this analysis, $n=1000$ squares were used for each image. If particles (MWCNT-rich domains) were found to lie within a square, the square size was decreased and the process was iterated until a length was reached where the squares were "empty" (pure epoxy with no visible MWCNT-rich domains). The size of the largest square that did not encompass MWCNT-containing pixels was defined as the FSL for that image. Thus, a sample with a lot of "empty" space had a large FSL, meaning a higher degree of aggregation or poorer dispersion. Unlike the domain radius, the FSL was clearly dependent on mass fraction, and slightly dependent on matrix cure temperature (Figure 7b). The FSL was fit with an exponential decay function, $f(x)=a \cdot\left(e^{(-b x)}\right)$; here, $x$ is the mass fraction, $a_{\left(T_{C}=25^{\circ} \mathrm{C}\right)}=(37.8 \pm 4.7), a_{\left(T_{C}=80^{\circ} \mathrm{C}\right)}=(42.1 \pm 5.6), b_{\left(T_{C}=25^{\circ} \mathrm{C}\right)}=$ $(1.68 \pm 0.63)$, and $b_{\left(T_{C}=80^{\circ} \mathrm{C}\right)}=(1.52 \pm 1.30)$ where uncertainties are determined from error propagation of the measured parameters and a $95 \%$ confidence interval on the model.

\subsection{Rheology Analysis}

One possible explanation for the drastic differences in DC conductivity for the two curing temperatures is the arrangement of carbon nanotube-rich domains; namely the domain size and relative locations with respect to one another. At the two curing temperatures used in this study, the polymer viscosity and motion of the nanofiller within the curing polymer matrix is expected to be different. The elevated curing temperature would likely give rise to a drop in viscosity at the early stages of cure and allow more MWCNT diffusion. At $\mathrm{T}_{\mathrm{c}}=80^{\circ} \mathrm{C}$, the individually dispersed nanotubes could diffuse farther, leading to association of single tubes into MWCNT-rich domains and more clustering of the domains with one another. If there is more aggregation or, alternatively, poorer dispersion in the $\mathrm{T}_{\mathrm{c}}=80{ }^{\circ} \mathrm{C}$ cured samples, then the domain-to-domain electrical conductive pathways would be more pronounced and result in a larger bulk conductivity for a given MWCNT concentration. To determine the diffusion coefficient of the domains during curing, rheological studies were performed on the thermosetting epoxy at both curing temperatures.

At $\mathrm{T}_{\mathrm{c}}=80^{\circ} \mathrm{C}$, the viscosity of the epoxy dropped at early times in the cure schedule and then increased rapidly as chemical crosslinking of the matrix occurred (Figure 8a) . 
This initial viscosity drop was not observed for the $\mathrm{T}_{\mathrm{c}}=25^{\circ} \mathrm{C}$ testing condition and a longer amount of time was required before a significant increase in the viscosity of the material was observed.

To approximate the mobility of the MWCNT-rich domains during curing of the epoxy, the Stokes-Einstein relationship[54] was used to calculate the diffusivity, $D$, for the two different cure temperatures:

$$
D=\frac{k_{B} T}{6 \pi \eta r}
$$

where $k_{B}$ is the Boltzmann constant, $T$ is the temperature, $\eta$ is the dynamic viscosity and $r$ is the diffusing object's radius (e.g., MWCNT-rich domain). It is acknowledged that this is a very rough approximation given that this relationship is meant to be applied to rigid spherical particles in low Reynolds number fluids. However, the aspect ratio of the domains was close to unity $\left(\mathrm{AR}_{\text {mode }}=1.55 \pm 0.29\right)$ and the objective here was to compare the relative diffusivity of the domains at the two different cure temperatures. Further, by rearranging this relationship to calculate the value of $D * r$, the diffusivity can be plotted as a function of cure time using the instantaneous values of $T$ and $\eta$ measured by shear rheology (Figure $8 \mathrm{~b}$ ) without assuming a value for the radius of the diffusing objects. The area under each curve is an estimate of the particle mobility for each cure temperature $\left(\mathrm{A}_{\mathrm{T}=25^{\circ} \mathrm{C}}=21.4 \times 10^{-18} \mathrm{~m}^{3}\right.$ and $\mathrm{A}_{\mathrm{T}=80}{ }^{\circ} \mathrm{C}=27.6 \times 10^{-18} \mathrm{~m}^{3}$ ). A ratio of these two values implies that domains within the samples prepared at $\mathrm{T}_{\mathrm{c}}=80{ }^{\circ} \mathrm{C}$ have roughly $25 \%$ more mobility during curing than those prepared at $\mathrm{T}_{\mathrm{c}}=25^{\circ} \mathrm{C}$. It appears that this modest increase in mobility allows increased network formation of MWCNT-rich domains .[55] The different diffusion behaviors likely account for the measured electrical conductivity differences as the MWCNT are able to acheive higher densities in the elevated cure temperature samples.

\subsection{Microstructure Impact on Conductivity}

While domain radius was independent of MWCNT loading and curing temperature, the dispersion quality, given by the FSL, had a direct impact on the DC electrical properties (Figure 9a). The clustering of the domains led to higher electrical conductivity than those measured for more well dispersed (low FSL) isolated domains with the same mass fraction of MWCNT.[22] For very low loadings of MWCNT, the spacing between domains is large, thus electrons cannot pass from one bundle to the next through the insulating epoxy matrix, greatly decreasing the DC conductivity (Figure 9). However, at low to intermediate loadings, the aggregation of domains appears to form an electrically percolating network.

Davis et al. 
A similar relationship is not observed between the AC conductivity and the FSL (Figure 9). The microwave spectroscopy employed here measures the dielectric changes within the individual MWCNT-rich domains.[37] Thus, the lack of correlation between the AC conductivity and the dispersion of the domains is to be expected. Rather, the difference between the two curing temperatures in terms of the AC conductivity (Figure $3 \mathrm{c}$ ) can be better explained by the decrease in viscosity during curing at elevated temperatures. A lower viscosity at the beginning of the cure cycle would allow tubes within a given domain the mobility to associate more closely with one another, forming stronger tube-tube connections.

Future work will address this formation of intimate tube-tube contacts between individual MWCNT. Small differences in the diffusion coefficient likely account for the dramatic difference in the electrical conductivity saturation plateau observed in the DC conductivity (Figure 3a) as nanoscopic gaps between nanotubes at higher cure temperatures would have more mobility available to form tube-tube contacts on a length scale not thoroughly imaged in this study.[56] The TEM images included in the Supporting Information provide some interesting initial indications of this phenomenon.

\section{Conclusion}

Through this investigation, the effects of mass fraction and matrix cure temperature on MWCNT-rich domain formation and aggregation and the resulting direct and alternating current electrical properties were explored. As the mass fraction of MWCNT was increased, the resulting nanocomposite samples were more electrically conducting. The DC conductivity of sample sets cured at two different temperatures varied by an order of magnitude after the percolation threshold was reached. In an attempt to determine a possible explanation for this drastic difference, the microstructure of the composite materials was investigated. While the MWCNT-rich domain size was independent of matrix cure temperature and MWCNT concentration, the domain separation or orientation (characterized by the free space length) decreased with increasing MWCNT concentration. The FSL was also slightly greater for the higher matrix cure temperature at each concentration. This slight variation in FSL is believed to be due to the difference in the diffusivity of the domains under the two different epoxy curing conditions. Based on the curing epoxy rheology and a simplistic application of Stokes-Einstein diffusion principles, it is expected that there would be $25 \%$ more domain mobility during curing in the nanocomposites cured at $\mathrm{T}_{\mathrm{c}}=80{ }^{\circ} \mathrm{C}$ than those prepared at $\mathrm{T}_{\mathrm{c}}=25^{\circ} \mathrm{C}$.

Davis et al. 
The larger mobility or diffusivity in the $\mathrm{T}_{\mathrm{c}}=80{ }^{\circ} \mathrm{C}$ samples during curing indicates that there is the potential for more aggregation or association of domains with one another, which would give rise to concentrated clusters of connected or electrically networked MWCNT domains in a sea of MWCNT-depleted epoxy. The higher aggregation samples can effectively be considered as a two-phase polymer blend where one phase is comprised of a highly loaded nanocomposite with MWCNT-rich domains while the other phase is predominantly unfilled (or extremely low MWCNT-containing) epoxy. This "composite within a composite" microstructure was observed in nanocomposites with the highest conductivity, while discrete, isolated domains were observed in samples with the lowest conductivity. Thus, the highly filled nanocomposite phase forms a percolating network (where electrical percolation occurs by interaction between the MWCNT-rich domains rather than through a conductive path/network formed by individual MWCNT) through the bulk material. This particular microstructure would allow electrical percolation at MWCNT concentrations lower than theoretical values.

A key finding of this work is that perfectly homogeneous, well-dispersed MWCNT might not be the optimal nanocomposite morphology to exploit the unique electrical properties of MWCNT. This work indicates that a certain degree of MWCNT-rich domain formation and subsequent aggregation is helpful in increasing conductivity at low MWCNT loadings. This knowledge is important for the design of conductive nanocomposites in which precise control of the conductivity is required. A second key finding of this study was the role that processing conditions can play in determining the final functional properties of a nanocomposite. Such results could have far-reaching impacts in the aerospace and automotive industries and may influence the design and production of electrically conducting nanocomposites. In conclusion, two processing variables (MWCNT mass fraction and matrix curing temperature) were used to tune the electrical conductivity by controlling the domain spacing in MWCNT nanocomposites. In future studies, the role of nanotube quality, functionalization and low shear aggregation on the electrical properties of nanocomposites will be explored.

\section{Acknowledgment}

Research was supported by the National Institute of Standards and Technology (NIST) Nano EH\&S Initiative, the National Research Council Research Assistanceship Program (for C.S.D.), and the National Nanotechnology Initiative. Additional funding was provided 
by a Cooperative Research Agreement (CRA) between the University of Maryland and the NIST grant 70NANB10H193 (for C.J.L., K.A.T., and B.N.); by a CRA between Rice University and NIST grant 70NANB12H188 (for N.D.O.). J.W.G and J.W.W. thank AFOSR for funding (MIPR F4FGA04094G002). Sample preparation was performed at the United States Naval Academy with the assistance of Prof. Paul Trulove, Dr. Kurt Sweely and Dr. E. Kate Brown. We thank Dr. Truman Wilson for his help maintaining the lithium ion microscope during SLIM imaging. All measurements were conducted at the Center for Nanoscale Science and Technology, a user facility at NIST. This work was performed in collaboration with the Material Measurement Laboratory at NIST in support of the National Nanotechnology Initiative.

\section{References}

[1] M. De Volder, S.H. Tawfick, S.J. Park, D. Copic, Z. Zhao, W. Lu, et al., Diverse 3D microarchitectures made by capillary forming of carbon nanotubes., Adv. Mater. 22 (2010) 4384-9. doi:10.1002/adma.201001893.

[2] M.H. Al-Saleh, U. Sundararaj, Processing-microstructure-property relationship in conductive polymer nanocomposites, Polymer (Guildf). 51 (2010) 2740-2747. doi:10.1016/j.polymer.2010.03.022.

[3] L. Gao, T.-W. Chou, E.T. Thostenson, A. Godara, Z. Zhang, L. Mezzo, Highly conductive polymer composites based on controlled agglomeration of carbon nanotubes, Carbon N. Y. 48 (2010) 2649-2651. doi:10.1016/j.carbon.2010.03.027.

[4] W. Bauhofer, J.Z. Kovacs, A review and analysis of electrical percolation in carbon nanotube polymer composites, Compos. Sci. Technol. 69 (2009) 1486-1498. doi:10.1016/j.compscitech.2008.06.018.

[5] S.G. Miller, T.S. Williams, J.S. Baker, F. Solá, M. Lebron-Colon, L.S. McCorkle, et al., Increased Tensile Strength of Carbon Nanotube Yarns and Sheets through Chemical Modification and Electron Beam Irradiation., ACS Appl. Mater. Interfaces. 6 (2014) 6120-6. doi:10.1021/am4058277.

[6] R. Ramasubramaniam, J. Chen, H. Liu, Homogeneous carbon nanotube/polymer composites for electrical applications, Appl. Phys. Lett. 83 (2003) 2928-2930. doi:10.1063/1.1616976.

[7] S. Fan, M.G. Chapline, N.R. Franklin, T.W. Tombler, A.M. Cassell, H. Dai, SelfOriented Regular Arrays of Carbon Nanotubes and Their Field Emission Properties, Science (80-. ). 283 (1999) 512-514. doi:10.1126/science.283.5401.512.

[8] O. Breuer, U. Sundararaj, Big returns from small fibers: A review of polymer/carbon nanotube composites, Polym. Compos. 25 (2004) 630-645. doi:10.1002/pc.20058.

[9] M.F.L. De Volder, S.H. Tawfick, R.H. Baughman, a J. Hart, Carbon nanotubes: present and future commercial applications., Science (80-. ). 339 (2013) 535-539. doi:10.1126/science.1222453.

[10] S.H. Lee, E. Cho, S.H. Jeon, J.R. Youn, Rheological and electrical properties of

Davis et al. 
polypropylene composites containing functionalized multi-walled carbon nanotubes and compatibilizers, Carbon N. Y. 45 (2007) 2810-2822.

doi:10.1016/j.carbon.2007.08.042.

[11] P. Pötschke, M. Abdel-Goad, I. Alig, S.M. Dudkin, D. Lellinger, Rheological and dielectrical characterization of melt mixed polycarbonate-multiwalled carbon nanotube composites, Polymer (Guildf). 45 (2004) 8863-8870.

doi:10.1016/j.polymer.2004.10.040.

[12] P. Pötschke, S.M. Dudkin, I. Alig, Dielectric spectroscopy on melt processed polycarbonate-multiwalled carbon nanotube composites, Polymer (Guildf). 44 (2003) 5023-5030. doi:10.1016/S0032-3861(03)00451-8.

[13] S. Torquato, Random Heterogeneous Media: Microstructure and Improved Bounds on Effective Properties, Appl. Mech. Rev. 44 (1991) 37-76. doi:10.1115/1.3119494.

[14] A. Allaoui, S. Bai, H. Cheng, J. Bai, Mechanical and electrical properties of a MWNT/epoxy composite, Compos. Sci. Technol. 62 (2002) 1993-1998. http://www.sciencedirect.com/science/article/pii/S026635380200129X (accessed September 1, 2014).

[15] Z. Spitalsky, D. Tasis, K. Papagelis, C. Galiotis, Carbon nanotube-polymer composites: Chemistry, processing, mechanical and electrical properties, Prog. Polym. Sci. 35 (2010) 357-401. doi:10.1016/j.progpolymsci.2009.09.003.

[16] N. Hu, Z. Masuda, C. Yan, G. Yamamoto, H. Fukunaga, T. Hashida, The electrical properties of polymer nanocomposites with carbon nanotube fillers, Nanotechnology. 19 (2008) 215701-10. doi:10.1088/0957-4484/19/21/215701.

[17] J.Z. Kovacs, B.S. Velagala, K. Schulte, W. Bauhofer, Two percolation thresholds in carbon nanotube epoxy composites, Compos. Sci. Technol. 67 (2007) 922-928. doi:10.1016/j.compscitech.2006.02.037.

[18] J. Obrzut, J. Douglas, S. Kharchenko, K. Migler, Shear-induced conductor-insulator transition in melt-mixed polypropylene-carbon nanotube dispersions, Phys. Rev. B Condens. Matter Mater. Phys. 76 (2007) 195420-9. doi:10.1103/PhysRevB.76.195420.

[19] R.-B. Yang, W.-S. Kuo, H.-C. Lai, Effect of carbon nanotube dispersion on the complex permittivity and absorption of nanocomposites in 2-18 GHz ranges, J. Appl. Polym. Sci. 131 (2014) 40963-7. doi:10.1002/app.40963.

[20] J. Sandler, M.S.P. Shaffer, T. Prasse, W. Bauhofer, K. Schulte, A.H. Windle, Development of a dispersion process for carbon nanotubes in an epoxy matrix and the resulting electrical properties, Polymer (Guildf). 40 (1999) 5967-5971. doi:10.1016/S0032-3861(99)00166-4.

[21] F. Vargas-Lara, J.F. Douglas, Confronting the complexity of CNT materials, Soft Matter. 11 (2015) 4888-4898. doi:10.1039/C5SM00912J.

[22] C.A. Martin, J.K.W. Sandler, M.S.P. Shaffer, M.-K. Schwarz, W. Bauhofer, K. Schulte, et al., Formation of percolating networks in multi-wall carbon-nanotubeepoxy composites, Compos. Sci. Technol. 64 (2004) 2309-2316. doi:10.1016/j.compscitech.2004.01.025.

[23] B. Vigolo, C. Coulon, M. Maugey, C. Zakri, P. Poulin, An experimental approach to the percolation of sticky nanotubes., Science (80-. ). 309 (2005) 920-923. 
doi:10.1126/science.1112835.

[24] M. Roy, J.K. Nelson, R.K. MacCrone, L.S. Schadler, C.W. Reed, R. Keefe, Polymer nanocomposite dielectrics-the role of the interface, IEEE Trans. Dielectr. Electr. Insul. 12 (2005) 629-643. doi:10.1109/TDEI.2005.1511089.

[25] N. Hu, Z. Masuda, G. Yamamoto, H. Fukunaga, T. Hashida, J. Qiu, Effect of fabrication process on electrical properties of polymer/multi-wall carbon nanotube nanocomposites, Compos. Part A Appl. Sci. Manuf. 39 (2008) 893-903. doi:10.1016/j.compositesa.2008.01.002.

[26] R. Krishnamoorti, Strategies for Dispersing Nanoparticles in Polymers, MRS Bull. 32 (2007) 341-347. doi:10.1557/mrs2007.233.

[27] M. Nadler, T. Mahrholz, U. Riedel, C. Schilde, A. Kwade, Preparation of colloidal carbon nanotube dispersions and their characterisation using a disc centrifuge, Carbon N. Y. 46 (2008) 1384-1392. doi:10.1016/j.carbon.2008.05.024.

[28] J.Z. Kovacs, K. Andresen, J.R. Pauls, C.P. Garcia, M. Schossig, K. Schulte, et al., Analyzing the quality of carbon nanotube dispersions in polymers using scanning electron microscopy, Carbon N. Y. 45 (2007) 1279-1288. doi:10.1016/j.carbon.2007.01.012.

[29] A. Lucas, C. Zakri, M. Maugey, M. Pasquali, P. Van Der Schoot, P. Poulin, Kinetics of Nanotube and Microfiber Scission under Sonication, J. Phys. Chem. C. 113 (2009) 20599-20605. doi:10.1021/jp906296y.

[30] F. Mirri, A.W.K. Ma, T.T. Hsu, N. Behabtu, S.L. Eichmann, C.C. Young, et al., Highperformance carbon nanotube transparent conductive films by scalable dip coating, ACS Nano. 6 (2012) 9737-9744. doi:10.1021/nn303201g.

[31] F.H. Gojny, J. Nastalczyk, Z. Roslaniec, K. Schulte, Surface modified multi-walled carbon nanotubes in CNT/epoxy-composites, Chem. Phys. Lett. 370 (2003) 820 824. doi:10.1016/S0009-2614(03)00187-8.

[32] Z.-M. Dang, L. Wang, Y. Yin, Q. Zhang, Q.-Q. Lei, Giant Dielectric Permittivities in Functionalized Carbon-Nanotube/ Electroactive-Polymer Nanocomposites, Adv. Mater. 19 (2007) 852-857. doi:10.1002/adma.200600703.

[33] J. Zhu, J. Kim, H. Peng, J.L. Margrave, V.N. Khabashesku, E. V. Barrera, Improving the Dispersion and Integration of Single-Walled Carbon Nanotubes in Epoxy Composites through Functionalization, Nano Lett. 3 (2003) 1107-1113. doi:10.1021/n10342489.

[34] F. Smits, Measurement of Sheet Resistivities with the Four-Point Probe, Bell Syst. Tech. J. May (1958) 711-718. http://onlinelibrary.wiley.com/doi/10.1002/j.15387305.1958.tb03883.x/abstract (accessed September 18, 2014).

[35] D.J. Yang, S.G. Wang, Q. Zhang, P.J. Sellin, G. Chen, Thermal and electrical transport in multi-walled carbon nanotubes, Phys. Lett. A. 329 (2004) 207-213. doi:10.1016/j.physleta.2004.05.070.

[36] Z. Wu, Z. Chen, X. Du, J.M. Logan, J. Sippel, M. Nikolou, et al., Transparent, conductive carbon nanotube films, Science (80-. ). 305 (2004) 1273-1276. doi:10.1126/science.1101243.

[37] N.D. Orloff, J. Obrzut, C.J. Long, T. Lam, P. Kabos, D.R. Novotny, et al., Dielectric Characterization by Microwave Cavity Perturbation Corrected for Nonuniform

Davis et al. 
Fields, IEEE Trans. Microw. Theory Tech. 62 (2014) 2149-2159.

doi:10.1109/TMTT.2014.2336775.

[38] A. Uhlir, The Potentials of Infinite Systems of Sources and Numerical Solutions of Problems in Semiconductor Engineering, Bell Syst. Tech. J. 34 (1955) 105-128. doi:10.1002/j.1538-7305.1955.tb03765.x.

[39] K.A. Twedt, L. Chen, J.J. Mcclelland, Scanning ion microscopy with low energy lithium ions, Ultramicroscopy. 142 (2014) 24-31.

doi:10.1016/j.ultramic.2014.03.014.

[40] C.J. Long, N.D. Orloff, K.A. Twedt, T. Lam, F. Vargas-Lara, M. Zhao, et al., Giant surface conductivity enhancement in a carbon nanotube composite by ultraviolet light exposure, Submitted. (2015).

[41] Z.Q. Zeng, a. Podpirka, S.W. Kirchoefer, T.J. Asel, L.J. Brillson, Direct correlation and strong reduction of native point defects and microwave dielectric loss in airannealed (Ba,Sr)TiO3, Appl. Phys. Lett. 106 (2015) 182903. doi:10.1063/1.4919891.

[42] D. Nuzhnyy, M. Savinov, V. Bovtun, M. Kempa, J. Petzelt, B. Mayoral, et al., Broadband conductivity and dielectric spectroscopy of composites of multiwalled carbon nanotubes and poly(ethylene terephthalate) around their low percolation threshold., Nanotechnology. 24 (2013) 055707-9. doi:10.1088/0957-4484/24/5/055707.

[43] D.J. Griffiths, An Introduction to Electrodynamics: From the Standpoint of the Electron Theory, 1922. doi:10.1038/110509b0.

[44] M. Zhao, K.A. Twedt, J.J. Mcclelland, J.A. Liddle, Imaging of Carbon Nanotubes Embedded in Polymer Composites via Low Energy Scanning Electron Microscopy and Scanning Lithium Ion Microscopy, Microsc. Microanal. 21 (2015) 513-514. doi:10.1017/S1431927615003360.

[45] M. Foygel, R.D. Morris, D. Anez, S. French, V.L. Sobolev, Theoretical and computational studies of carbon nanotube composites and suspensions: Electrical and thermal conductivity, Phys. Rev. B - Condens. Matter Mater. Phys. 71 (2005) 104201-8. doi:10.1103/PhysRevB.71.104201.

[46] R. Haggenmueller, C. Guthy, J.R. Lukes, J.E. Fischer, K.I. Winey, Single Wall Carbon Nanotube / Polyethylene Nanocomposites : Thermal and Electrical Conductivity, Macromolecules. 40 (2007) 2417-2421.

[47] T. Xiao, H.L. Yang, G.P. Zhang, The influence of carbon nanotube structure on complex permittivity and determination of filler density by microwave techniques, $\mathrm{J}$. Appl. Phys. 110 (2011). doi:10.1063/1.3599857.

[48] N.M. Barkoula, B. Alcock, N.O. Cabrera, T. Peijs, Fatigue properties of highly oriented polypropylene tapes and all-polypropylene composites, Polym. Polym. Compos. 16 (2008) 101-113. doi:10.1002/pc.

[49] N. George, J.C. C.S., A. Mathiazhagan, R. Joseph, High performance natural rubber composites with conductive segregated network of multiwalled carbon nanotubes, Compos. Sci. Technol. 116 (2015) 33-40. doi:10.1016/j.compscitech.2015.05.008.

[50] N. Otsu, A Threshold Selection Method from Gray-Level Histograms, IEEE Trans. Syst. Man, Cypernetics. 9 (1979) 62-66.

[51] E.R. Weibel, G.S. Kistler, W.F. Scherle, Practical stereological methods for morphometric cytology, J. Cell Biol. 30 (1966) 23-38. doi:10.1083/jcb.30.1.23.

Davis et al. 
[52] D. Freedman, P. Diaconis, On the histogram as a density estimator: L2 theory, Zeitschrift Für Wahrscheinlichkeitstheorie Und Verwandte Gebiete. 57 (1981) 453 476. doi:10.1007/BF01025868.

[53] H.S. Khare, D.L. Burris, A quantitative method for measuring nanocomposite dispersion, Polymer (Guildf). 51 (2010) 719-729. doi:10.1016/j.polymer.2009.12.031.

[54] J.T. Edward, Molecular volumes and the Stokes-Einstein equation, J. Chem. Educ. 47 (1970) 261-270. doi:10.1021/ed047p261.

[55] N. Kiuna, C.J. Lawrence, Q.P.. Fontana, P.D. Lee, T. Selerland, P.D.M. Spelt, A model for resin viscosity during cure in the resin transfer moulding process, Compos. Part A Appl. Sci. Manuf. 33 (2002) 1497-1503. doi:10.1016/S1359835X(02)00177-X.

[56] K.L. Lasater, E.T. Thostenson, In situ thermoresistive characterization of multifunctional composites of carbon nanotubes, Polymer (Guildf). 53 (2012) 53675374. doi:10.1016/j.polymer.2012.09.022. 


\begin{tabular}{|c|c|c|c|c|}
\hline \multicolumn{5}{|c|}{ Direct Current (DC) Conductivity by Percolation Theory } \\
\hline & $\sigma_{c}$ & $\phi_{v, c}$ & $\mathrm{t}$ & $\mathrm{R}^{2}$ \\
\hline $\mathrm{T}_{\mathrm{c}}=25^{\circ} \mathrm{C}, \sigma_{\mathrm{DC}}$ & $0.0025 \pm 0.0002$ & $0.0547 \pm 0.0211$ & $1.195 \pm 0.633$ & 0.88 \\
\hline $\mathrm{T}_{\mathrm{c}}=80^{\circ} \mathrm{C}, \sigma_{\mathrm{DC}}$ & $0.0327 \pm 0.0120$ & $0.0393 \pm 0.0191$ & $1.642 \pm 0.794$ & 0.93 \\
\hline \multicolumn{5}{|c|}{ Alternating Current (AC) Conductivity by Saturation and Growth } \\
\hline & $\sigma_{c}^{A C}$ & $r$ & $\phi_{v, c}^{A C}$ & $\mathrm{R}^{2}$ \\
\hline $\mathrm{T}_{\mathrm{c}}=25^{\circ} \mathrm{C}, \sigma_{\mathrm{AC}}$ & $0.30 \pm 0.01$ & $6.18 \pm 1.02$ & $0.36 \pm 0.02$ & 0.99 \\
\hline $\mathrm{T}_{\mathrm{c}}=80^{\circ} \mathrm{C}, \sigma_{\mathrm{AC}}$ & $0.35 \pm 0.02$ & $6.30 \pm 1.09$ & $0.37 \pm 0.04$ & 0.99 \\
\hline
\end{tabular}

Table 1: Fitting parameter values for two functions fit to the conductivity data. The percolation theory exponential function has been applied to DC conductivity data as a function of volume fraction $\%$ of MWCNT. An exponential saturation and growth function has been applied to the AC conductivity data as a function of volume fraction \% of MWCNT. A logistic growth model was used to fit the AC conductivity behavior in order to qaulititively understand the difference between the cure temperatures. Uncertainty values represent a $95 \%$ confidence interval and the $\mathrm{R}^{2}$ value indicates the goodness of fit using a bisquare regression function. 


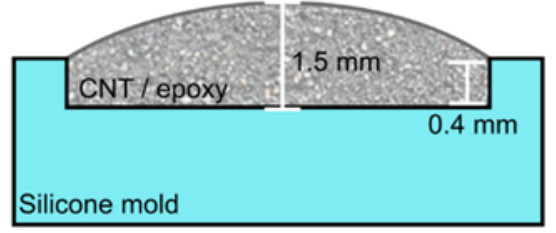

b) Top and bottom of sample removed

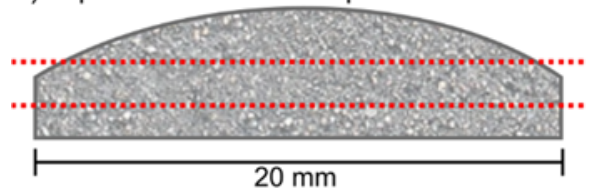

c) Gradient thickness ends removed

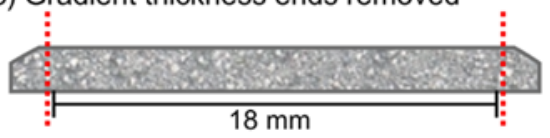

d) Final sample for DC and AC conductivity

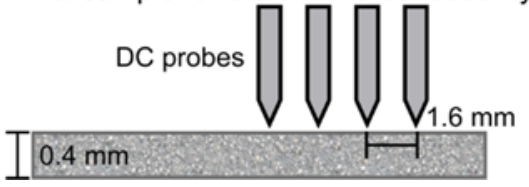

a) Liquid MWCNT/epoxy poured in mold

Figure 1: Schematic of sample preparation for electrical conductivity testing. a) Liquid MWCNT/epoxy mixture poured into silicone mold. Excess material was added to each cavity of the mold to prevent dewetting. Significant curvature due to surface tension was observed on the top surface of each sample. b-d) Subsequent sectioning of the cured MWCNT composite sample to a final size of $0.4 \mathrm{~mm} \times 3 \mathrm{~mm} \times 18 \mathrm{~mm}$ ( standard deviation $=0.04 \mathrm{~mm}$ for all three dimensions). Dashed lines indicate locations where material was removed, either by grinding (b) or direct sectioning (c and d). d) Probe-based direct current conductivity measurements were performed at several locations on each sample with care being taken to stay relatively close to the center of the sample in both lateral directions. AC conductivity of samples was also measured in this geometry by microwave cavity perturbation (described in subsequent sections).

a) SLIM sample preparation by microtome

b) SLIM experimental setup
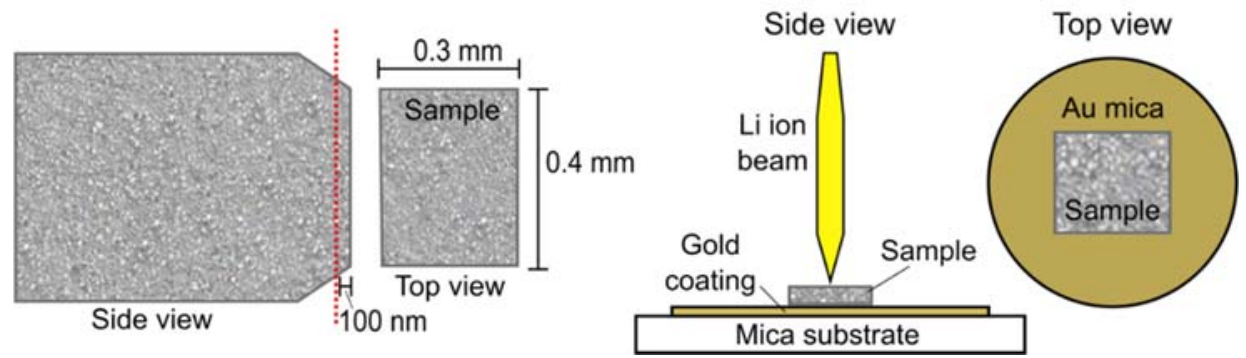

Figure 2: Schematic of SLIM sample preparation. a) Dashed red line indicates location of microtome sectioning. Films $(t=100 \mathrm{~nm})$ were prepared by microtome and placed onto freshly-cleaved mica substrates sputter coated with gold. b) Samples on conductive substrates were imaged with SLIM. Both the side view (left) and top view (right) of the SLIM sample configuration are shown. 

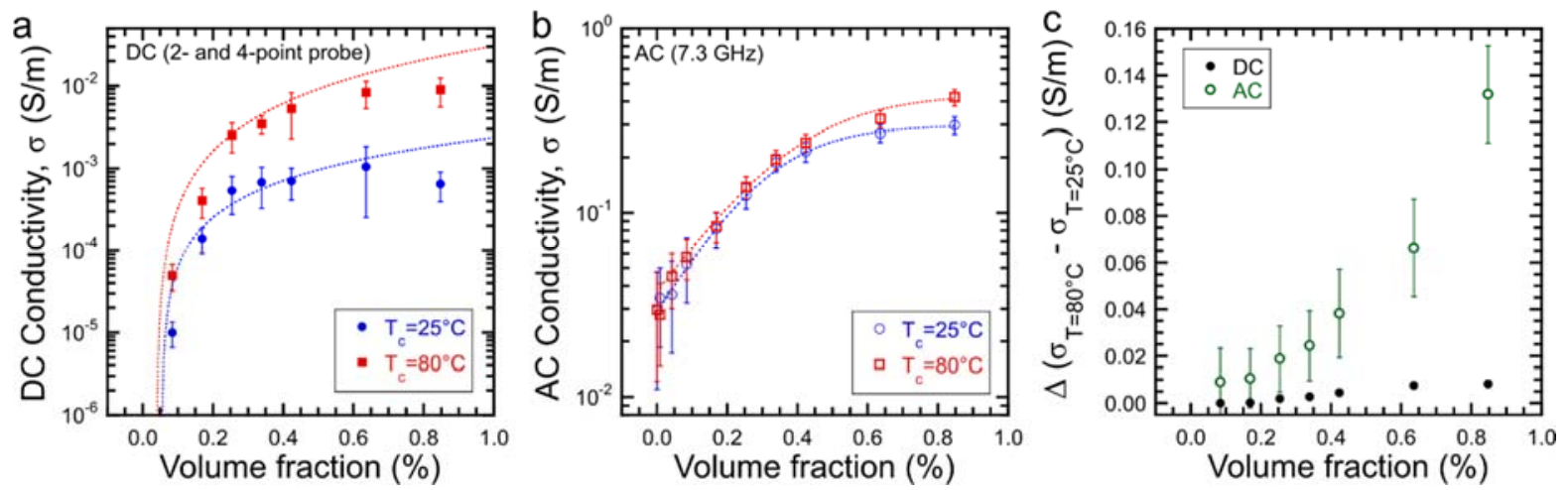

Figure 3: Electrical properties (both DC and AC) scale with MWCNT concentration, shown here as conductivity $(\mathrm{S} / \mathrm{m})$ versus volume fraction $\%$ of MWCNT. The DC conductivity (a) was determined by four-point probe measurements for higher conductivity materials and two-point probe for lower conductivity materials and the AC conductivity (b) was measured by noncontact microwave cavity perturbation measurements. Two sample sets prepared at $\mathrm{T}_{\mathrm{c}}=25^{\circ} \mathrm{C}$ and $\mathrm{T}_{\mathrm{c}}=80^{\circ} \mathrm{C}$ were tested for both $\mathrm{DC}$ and $\mathrm{AC}$ conductivity. The dashed lines are the percolation theory (DC conductivity) and saturation and growth (AC conductivity) fits described in the text (blue line is $\mathrm{T}_{\mathrm{c}}=25^{\circ} \mathrm{C}$, red line is $\mathrm{T}_{\mathrm{c}}=80^{\circ} \mathrm{C}$ ). Note the different values on the y-axes of both graphs. The vertical bars represent one standard deviation of multiple measurements conducted on each sample. The difference in the average conductivity of samples prepared at the two curing temperatures is shown (c) with the error bars representing the standard error.

Davis et al. 

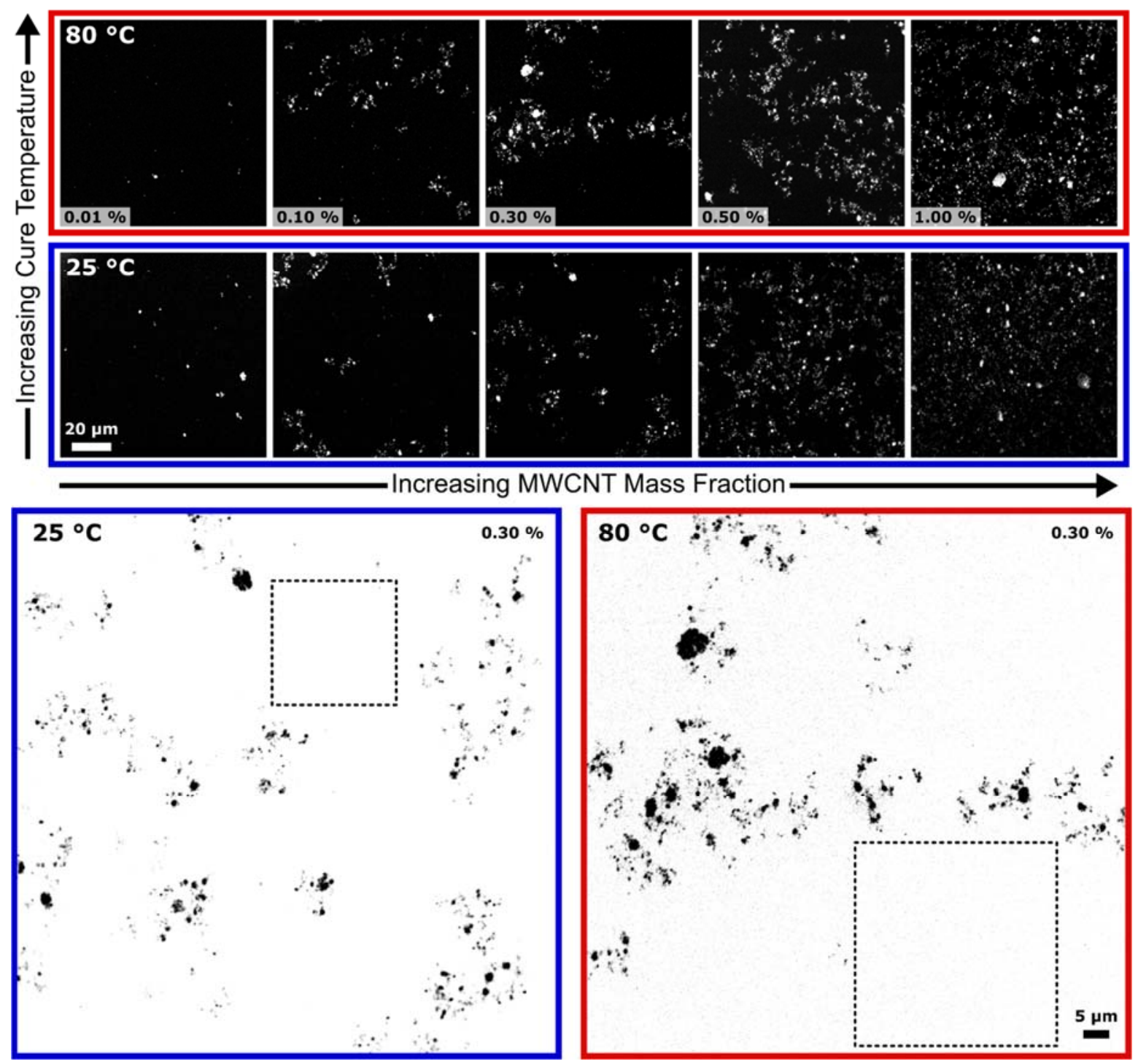

Figure 4: Binarized SLIM images of MWCNT/epoxy nanocomposites with increasing concentration ("\%" indicates mass fraction \% of MWCNT). Nanocomposites prepared at $\mathrm{T}_{\mathrm{c}}=25^{\circ} \mathrm{C}$ (blue box) and $\mathrm{T}_{\mathrm{c}}=80^{\circ} \mathrm{C}$ (red box). White areas in images are MWCNT-rich domains while the black areas are epoxy-rich portions. Scale bar in bottom left image applies to all small images. Larger images in bottom row are of 0.30 mass fraction $\%$ of MWCNT prepared at both temperatures. Color has been inverted to allow MWCNT-rich domains (now in black) to be more easily observed. Dashed squares represent average FSL determined for each image $\left(\mathrm{FSL}_{\mathrm{T}=25^{\circ} \mathrm{C}}=25.5 \mu \mathrm{m}\right.$ and $\left.\mathrm{FSL}_{\mathrm{T}}=80^{\circ} \mathrm{C}=40.5 \mu \mathrm{m}\right)$. Scale bar in lower right corner applies to both large images.

Davis et al. 


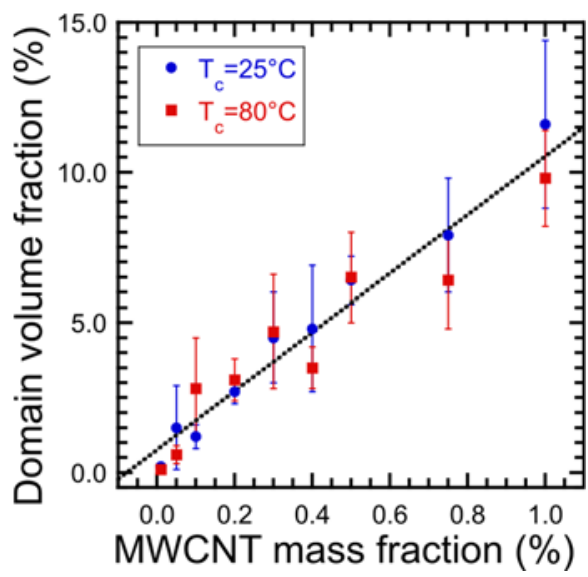

Figure 5: The domain volume fraction scales linearly with the mass fraction of MWCNT. The dashed line is a linear fit with a slope of $9.76 \pm 1.37$ where the uncertainty represents a $95 \%$ confidence interval. The vertical error bars represent one standard deviation of measurements performed on multiple images from the same sample.
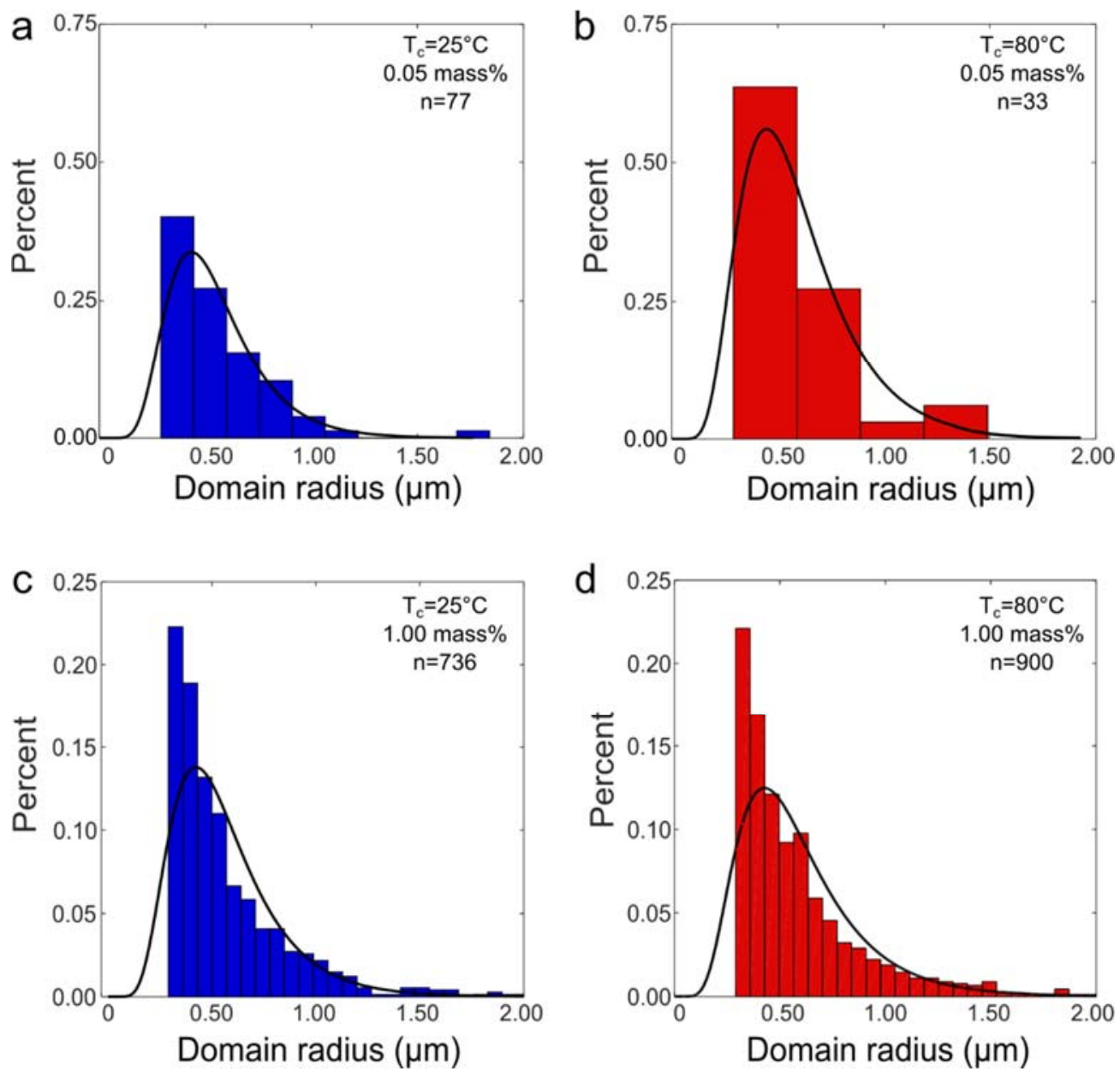

Figure 6: MWCNT-rich domain radius at two cure temperatures and MWCNT concentrations. The mode of the domain radius was independent of MWCNT mass fraction (0.05 mass\% (a-b) and 1.00 mass\% (c-d)). The black curve on each histogram is the log- 
normal probability distribution function calculated for each sample. Note that there is an artificial cutoff in the data for domain radii smaller than $0.125 \mu \mathrm{m}$ due to the image analysis constraint described in the text.

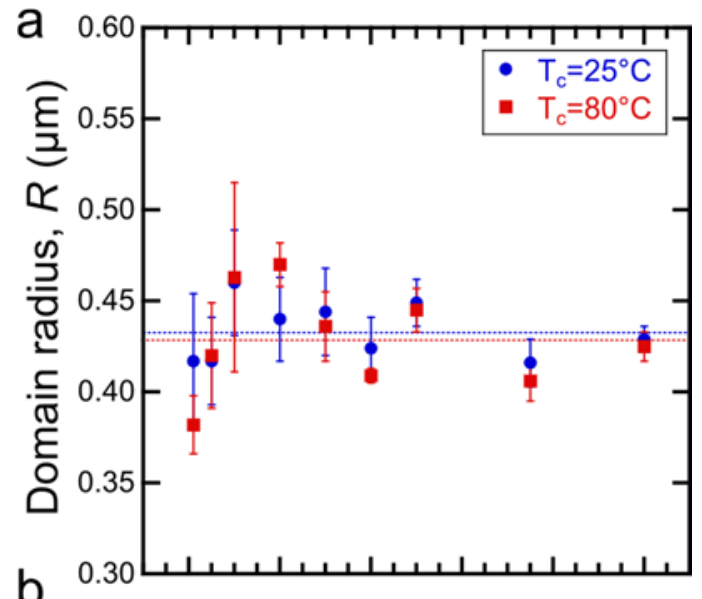

Figure 7: (a) Domain radius and (b) free space length, FSL, as a function of the mass fraction of MWCNT. The legend in a) and $\mathrm{x}$-axis in b) apply to both plots. The thin dashed horizontal lines in (a) represent the average of the mode domain radii. The dashed lines in (b) are first-order exponential fits (line colors correspond to cure temperatures: $\mathrm{T}_{\mathrm{c}}=25^{\circ} \mathrm{C}$ (blue) and $\mathrm{T}_{\mathrm{c}}=80{ }^{\circ} \mathrm{C}$

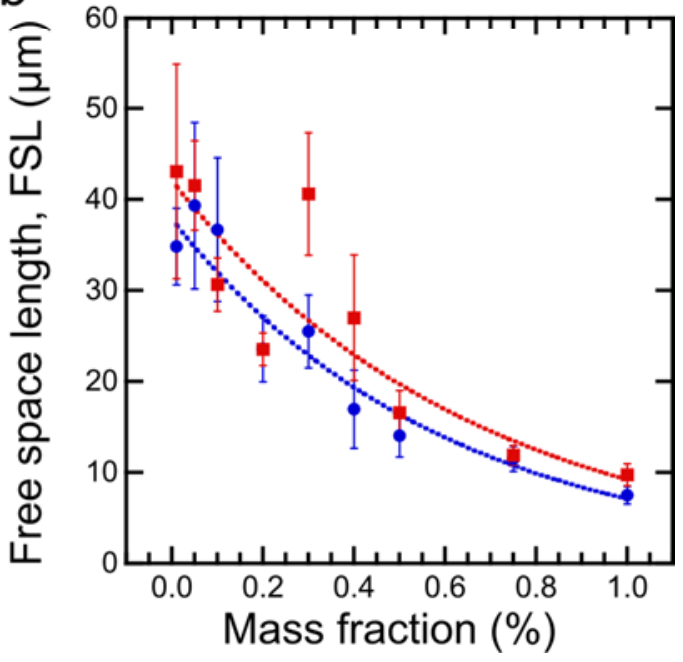
(red)). Error bars represent one standard deviation of all domain radii measured for each respective MWCNT mass fraction and matrix cure temperature (a) and for multiple applications of the FSL macro (1000 squares per application) (b). 

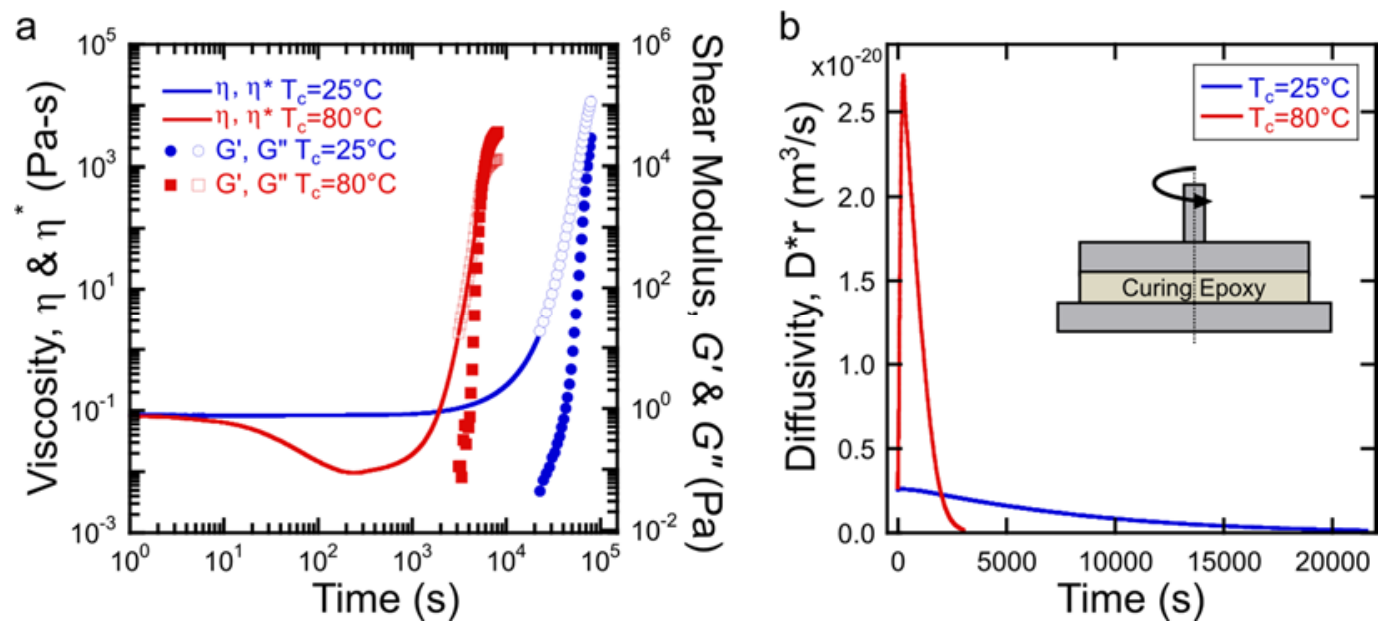

Figure 8: Estimation of particle diffusivity by rheological characterization of epoxy samples during curing. a) Parallel plate rheology study of epoxy formulation used in the present study while curing at $\mathrm{T}_{\mathrm{c}}=25^{\circ} \mathrm{C}$ (blue) or $\mathrm{T}_{\mathrm{c}}=80^{\circ} \mathrm{C}$ (red). Solid lines represent simple, $\eta$, (steady shear) and complex, $\eta^{*}$, (oscillatory shear) viscosity data while the closed and open symbols represent the shear storage, G', and loss moduli, G', respectively. b) The diffusivity was calculated using the Stokes-Einstein relationship over time for both cure temperatures. The inset is a schematic representation of the experimental setup.

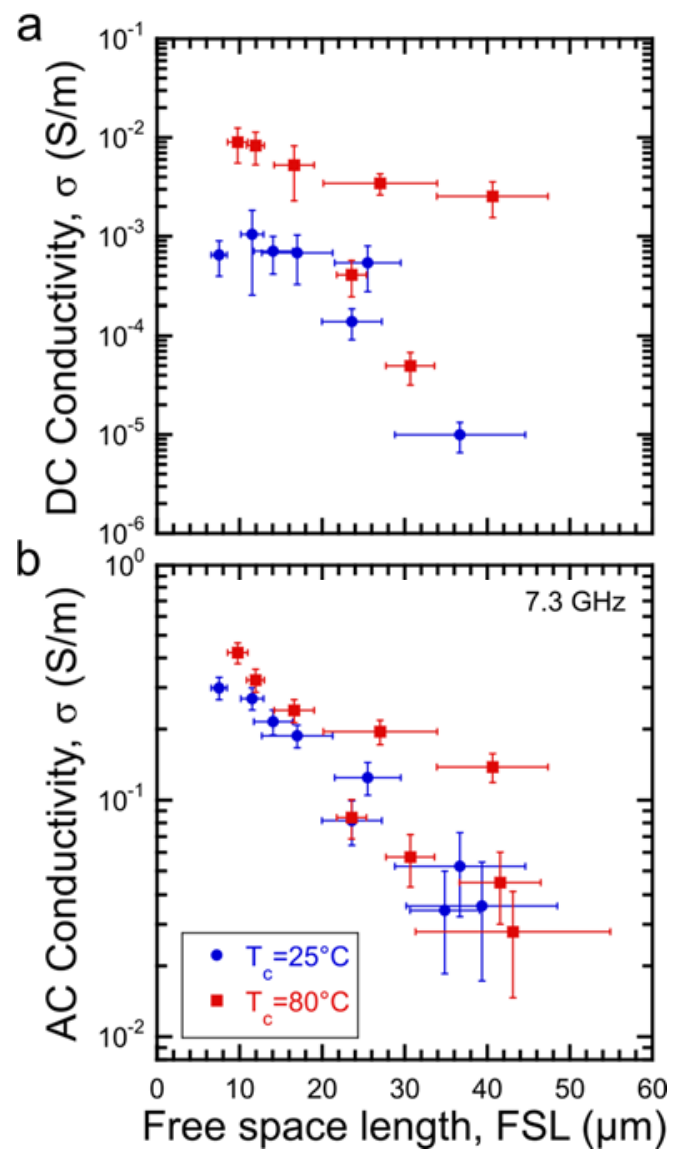

Davis et al. 
Figure 9: Electrical properties scale with MWCNT domain morphology. Conductivity $(\mathrm{S} / \mathrm{m})$ decreases with increasing domain spacing as indicated by the free space length (note relative scales of y-axes). Legend in (b) applies to both plots. Error bars represent one standard deviation of multiple measurements performed on each sample. 